\title{
Gauge string in the fermion asymmetric matter.
}

\author{
A.A. Kozhevnikov \\ Laboratory of Theoretical Physics, \\ S.L. Sobolev Institute for Mathematics, \\ 630090, Novosibirsk-90, Russian Federation *
}

(April 4, 2018)

\begin{abstract}
Two new effects of interaction of the gauge string with a homogeneous density of fermions are considered in a gauge model with an anomalous coupling of vector fields with fermions. First, the presence of an induced nonzero magnetic-like helicity on the straight string is demonstrated. Second, it is shown that the equation of motion of the string is modified by a nonlinear term that can be decomposed into the correction to the string tension and an additional force perpendicular to the tangent and normal vectors of the string. Static configurations are found and their stability is studied.
\end{abstract}

PACS number(s): $11.27+\mathrm{d}, 98.80 . \mathrm{Cq}$

*Electronic address: kozhev@math.nsc.ru 
The presence of a large asymmetry of some fermionic charges, in particular, the lepton number, constitutes an interesting theoretical possibility [1] 3]. Current experimental bounds on this type of asymmetry are large enough [4]. On the other hand, the stringlike defects analogous to those already observed in type II superconductors immersed into magnetic field, might have been produced at early epoch of the evolution of the Universe [5, [0]. A wide class of such defects is described as a classical solution to some gauge field theoretical models like an Abelian Higgs model (AHM) [7]. In particular, the Z string [8] is an embedding of the Abrikosov-Nielsen-Olesen solution [7] into $\mathrm{SU}(2) \times \mathrm{U}(1)$ electroweak model. It is important that the gauge field configuration of a $\mathrm{Z}$ string contributes [9] to the right hand side of the anomaly equation for the sum of the baryon and lepton numbers. The above developments raise the question of could a nonzero asymmetry of some fermionic charge exert an influence on the string motion?

Since the pioneering work [10] devoted to the derivation of the string equation of motion from AHM in the zero thickness limit, attempts were undertaken to obtain the finite thickness corrections to the string motion [11 13]. Here we will find the correction of basically different origin which arises due to the presence of a nonzero density of some fermionic charge characterized by a nonzero chemical potential, provided an underlying gauge model contains an anomalous interaction of gauge fields with chiral fermions. The latter takes place, for example, in the case of a $\mathrm{Z}$ string and the lepton and baryon numbers of the chiral leptons and quarks of the electroweak theory. The fact is that the action of the model should then contain an additional term

$$
\Delta S=\int d t \mu n_{\mathrm{CS}},
$$

[14, 15], where $\mu$ is the chemical potential of fermions, and $n_{\mathrm{CS}}$ is the Chern-Simons number of the gauge field. Hereafter the case of zero temperature is considered, $T=0$. Since some parity-non-invariant configurations of the gauge field trapped by the string are known to have a nonzero $n_{\mathrm{CS}}$ [9, 16, 17], the above term in the action should manifest itself in the string equation of motion. Below corresponding correction is found explicitly. As a by-product, the magnetic-like helicity induced on the straight gauge string by a nonzero fermionic density is evaluated.

In order the presentation to be self-contained, let us sketch the derivation of the string equation of motion from the action of AHM. The gauge coupling constant $g$, the self-coupling of the Higgs field $\lambda^{2}$, and its vacuum expectation value $\eta^{2} / 2$ are such that $m_{H} \gg m_{V}$; $\ln m_{H} / m_{V}$ is also large, where $m_{V}=g \eta$ and $m_{H}=\lambda \eta$ are the masses of the gauge and Higgs bosons, respectively. This is the London limit of AHM. In this limit, the Higgs field is $\phi=\eta \exp i \chi / \sqrt{2}$ everywhere except the string line where $|\phi|$ vanishes at the distance scale $\sim m_{H}^{-1}$, and $\chi$ is the spacetime dependent phase. Then the AHM action can be written as

$$
S=\int d^{4} x\left[-F_{\mu \nu}^{2} / 4+m_{V}^{2}\left(A_{\mu}+\partial_{\mu} \chi / g\right)^{2} / 2\right],
$$

where $A_{\mu}=\left(A_{0}, \mathbf{A}\right), F_{\mu \nu}=\partial_{\mu} A_{\nu}-\partial_{\nu} A_{\mu}$ being the vector potential and the field strength tensor, respectively. The contribution of the Higgs field potential is suppressed in this limit by the factor $1 / \ln m_{H} / m_{V} \ll 1$ as compared to the kinetic energy of this field, see below, and hence is neglected. Note that the case of Z-string is obtained by the replacement $A_{\mu} \rightarrow Z_{\mu}, g \rightarrow \sqrt{g^{2}+g^{\prime 2}} / 2$, where $Z_{\mu}$ is the vector potential of $Z$ boson, $g$ and $g^{\prime}$ are the 
$\mathrm{SU}(2)$ and $\mathrm{U}(1)$ coupling constants of the electroweak theory. The field equation for the vector potential obtained from the variation of the action (2) under the condition $\partial_{\mu} A^{\mu}=0$, is $\left(\partial^{2}+m_{V}^{2}\right) A_{\mu}=-m_{V}^{2} \partial_{\mu} \chi / g$. It is solved to give

$$
\begin{aligned}
A_{\mu}+\partial_{\mu} \chi / g & =\left[1-m_{V}^{2}\left(\partial^{2}+m_{V}^{2}\right)_{\mathrm{ret}}^{-1}\right] \partial_{\mu} \chi / g, \\
F_{\mu \nu} & =-m_{V}^{2}\left(\partial^{2}+m_{V}^{2}\right)_{\mathrm{ret}}^{-1} \partial_{[\mu} \partial_{\mu]} \chi,
\end{aligned}
$$

where the subscript points to the choice of the Green function as the retarded one. The expression for the commutator of derivatives of the singular phase of the Higgs field is [11]

$$
\partial_{[\mu} \partial_{\mu]} \chi=2 \pi \varepsilon_{\mu \nu \alpha \beta} \int d^{2} s \partial_{\tau} X^{\alpha} \partial_{\sigma} X^{\beta} \delta^{(4)}(x-X),
$$

where $X \equiv X(\sigma, \tau)$ is the string world sheet, $s^{A}=(\tau, \sigma)$ is the two-vector, and the unit winding number of the Higgs field is taken. In what follows, the space Fourier transforms of the gauge field configuration found earlier [18] is often used. This form is especially comfortable because the fields are expressed through elementary functions rather than the special ones. Using (4i) one can obtain the explicit form for the field strength tensor, the gauge invariant combination of the vector potential, and the vector $v_{\mu} \equiv \partial_{\mu} \chi / g$ as

$$
\begin{aligned}
F_{\mu \nu}(k) & =-\frac{2 \pi}{g} \varepsilon_{\mu \nu \alpha \beta} \int d^{2} s \partial_{\tau} X^{\alpha} \partial_{\sigma} X^{\beta} \frac{m_{V}^{2}}{m_{V}^{2}-k^{2}-i \varepsilon k^{0}} \exp (i k X), \\
\left(A_{\mu}+\partial_{\mu} \chi / g\right)(k) & =-\frac{k^{2}}{m_{V}^{2}-k^{2}-i \varepsilon k^{0}} v_{\mu}(k), \\
v_{\mu}(k) & =-\frac{2 \pi i}{g} \varepsilon_{\mu \nu \alpha \beta} \frac{k^{\nu}}{k^{2}} \int d^{2} s \partial_{\tau} X^{\alpha} \partial_{\sigma} X^{\beta} \exp (i k X) .
\end{aligned}
$$

In the case of many contours one should sum over all of them. The insertion of Eq. (5) into Eq. (2) gives the gauge vortex string action in the London limit. One can observe that, similar to the potential energy of the Higgs field, the contribution of the gauge field strength to the action is suppressed, in this limit, by the same factor $1 / \ln m_{H} / m_{V} \ll 1$. This is due to the $k^{\nu}$ factor in the numerator of the expression for the gauge invariant combination of the vector potential. Then the dominant contribution to the action becomes

$$
\begin{aligned}
S= & -\frac{m_{V}^{2}}{2}\left(\frac{2 \pi}{g}\right)^{2} \int \frac{d^{4} k}{(2 \pi)^{4}} \frac{k^{2}}{\left(m_{V}^{2}-k^{2}\right)^{2}+\left(\varepsilon k^{0}\right)^{2}} \int d^{2} s_{1} d^{2} s_{2} \exp \left(i k X_{12}\right) \\
& \times\left[\left(\dot{X}_{1} \dot{X}_{2}\right)\left(X_{1}^{\prime} X_{2}^{\prime}\right)-\left(\dot{X}_{1} X_{2}^{\prime}\right)\left(\dot{X}_{2} X_{1}^{\prime}\right)\right],
\end{aligned}
$$

where $X_{1,2} \equiv X\left(\sigma_{1,2}, \tau_{1,2}\right)$. Hereafter the prime over $X$ denotes the derivative with respect to the parameter along the contour, the overdot denotes the derivative with respect to (proper)time. Since the remaining logarithmic divergence at coincident $s_{1}^{A}=s_{2}^{A}$ is due to our ignorance of the Higgs field profile at the distances smaller than $m_{H}^{-1}$, equivalently, at the momenta larger than $m_{H}$, we insert the form factor $m_{H}^{2} /\left(m_{H}^{2}-k^{2}\right)$ into the integrand of Eq. (6), in order to take into account the above feature of the Higgs field. The detailed form of the cutoff factor is irrelevant within the logarithmic accuracy adopted here. Using the representation $\left(m^{2}-k^{2}-i \varepsilon\right)^{-1}=i \int_{0}^{\infty} d \alpha \exp \left[i \alpha\left(k^{2}-m^{2}+i \varepsilon\right)\right]$, one can integrate over $k$ to obtain the regularized action 


$$
\begin{aligned}
S_{\mathrm{reg}}= & -\frac{m_{V}^{2}}{8 g^{2}} \int_{0}^{\infty} \frac{d \alpha}{\alpha^{2}} e^{-\varepsilon \alpha}\left(e^{-i \alpha m_{H}^{2}}-e^{-i \alpha m_{V}^{2}}\right) \\
& \times \int d^{2} s_{1} d^{2} s_{2} e^{-i X_{12}^{2} / 4 \alpha}\left[\left(\dot{X}_{1} \dot{X}_{2}\right)\left(X_{1}^{\prime} X_{2}^{\prime}\right)-\left(\dot{X}_{1} X_{2}^{\prime}\right)\left(\dot{X}_{2} X_{1}^{\prime}\right)\right] .
\end{aligned}
$$

Since the dominant contribution comes from $s_{2}^{A}=s_{1}^{A}+z^{A}$ close to $s_{1}^{A}$, one may use the expansion $X_{12}^{2}=z^{A} z^{B} \partial_{A} X_{\mu} \partial_{B} X^{\mu}$ to integrate over $d^{2} s_{2}=d^{2} z$. The result is the action in the zeroth order in the vortex thickness, $S_{\text {reg }}=-\varepsilon_{\mathrm{v}} \int d^{2} s \sqrt{-X^{\prime 2} \dot{X}^{2}+\left(X^{\prime} \dot{X}\right)^{2}}$, with $\varepsilon_{\mathrm{v}}=$ $\pi \eta^{2} \ln m_{H} / m_{V}$ being the energy per unit length. This is the familiar Nambu-Goto form. 巴 As is known, in the physical transverse gauge $\tau=X^{0}, \dot{\mathbf{X}} \cdot \mathbf{X}^{\prime}=0, \mathbf{X}^{\prime 2}=1-\dot{\mathbf{X}}^{2}$, the equation of motion acquires the simple form

$$
\ddot{\mathbf{X}}-\mathbf{X}^{\prime \prime}=0 .
$$

However, in the situations when either the first stages of the string loop collapse are considered, or in the case of the long wave perturbations propagating on the string, or both, the transverse velocity is nonrelativistic. Then the retardation is inessential, and one can neglect $k^{0}$ in comparison with the large gauge boson mass $m_{V}$ in the propagator of Eq. (5). This permits one to integrate over $k^{0}$ to obtain the mixed, $(\mathbf{k}, t)$, Fourier representation for all necessary quantities. It is just this case that should be kept in mind in what follows.

Let us discuss the possible modifications of Eq. (8) by the presence of an induced ChernSimons (CS) term. The nonzero density of fermions provides the naturally preferable reference frame in which the fermionic sea is at rest. Then all the $3 \mathrm{D}$ vectors are taken relative to this reference frame. In principle, the presence of the CS term in the action, Eq. (1), disturbs the gauge field configuration of the string, see Ref. [19]. However, this disturbance does not alter the form of Eq. (8), provided the chemical potential is sufficiently small, namely, $\mu \ll m_{V}(2 \pi / g)^{2}$. Indeed, the field equation for the strength of magnetic field, in the presence of the CS term, becomes

$$
\boldsymbol{\nabla} \times \mathbf{H}=m_{V}^{2}\left(\frac{1}{g} \nabla \chi-\mathbf{A}\right)+\frac{\mu g^{2}}{2 \pi^{2}} \mathbf{H} .
$$

Hereafter the explicit dependence on time is omitted from all the notations. Then the Fourier components of the corrections to $\mathbf{H}$ and $(\boldsymbol{\nabla} \chi / g-\mathbf{A})$ that arise due to a nonzero chemical potential are found to be

$$
\begin{aligned}
\delta \mathbf{H}(\mathbf{k}) & =\frac{i \mu g^{2}}{2 \pi^{2}} \frac{[\mathbf{k} \times \mathbf{H}(\mathbf{k})]}{\mathbf{k}^{2}+m_{V}^{2}}, \\
\delta(\nabla \chi / g-\mathbf{A})(\mathbf{k}) & =\frac{\mu g^{2}}{2 \pi^{2}} \frac{\mathbf{H}(\mathbf{k})}{\mathbf{k}^{2}+m_{V}^{2}},
\end{aligned}
$$

where $\mathbf{H}(\mathbf{k})=2 \pi g^{-1} \int d \sigma \mathbf{X}^{\prime} \exp (-i \mathbf{k} \cdot \mathbf{X}) m_{V}^{2} /\left(\mathbf{k}^{2}+m_{V}^{2}\right)^{-1}$ is the unperturbed strength of magnetic field, while $A_{0}+\dot{\chi} / g$ remains unchanged. The leading correction is perpendicular

\footnotetext{
${ }^{1}$ The longitudinal correction to the electric field strength arising due to the time component of the vector potential was not properly taken into account in 18. By this reason the kinetic part of the action appeared there to be suppressed by some large factor.
} 
to unperturbed fields. The action being quadratic in the fields remains unchanged to first order in $\mu$. Interestingly enough, but even the mirror-invariant straight string acquires a nonzero magnetic-like helicity in the presence of a nonzero fermionic density characterized by $\mu \neq 0$. In fact, using Eq. (10), one finds the induced helicity to be

$$
h_{\text {induced }}=\int d^{3} x(\mathbf{H} \cdot \delta \mathbf{A}+\delta \mathbf{H} \cdot \mathbf{A})=\frac{\mu g^{2}}{\pi^{2}} \int \frac{d^{3} k}{(2 \pi)^{3}} \frac{|\mathbf{H}(\mathbf{k})|^{2}}{\mathbf{k}^{2}+m_{V}^{2}} .
$$

Since $\mathbf{H}(\mathbf{k})$ for the z-directed straight string is $\mathbf{H}(\mathbf{k})=(2 \pi)^{2} g^{-1} m_{V}^{2}\left(\mathbf{k}^{2}+m_{V}^{2}\right)^{-2} \delta\left(k_{z}\right) \mathbf{e}_{z}, \mathbf{e}_{z}$ being the unit vector in $\mathrm{z}$ direction, the induced helicity per unit length $L$ evaluated from Eq. (11) is $h_{\text {induced }} / L=\mu / 2 \pi$. Its contribution to the action is quadratic in $\mu$ and can be neglected under the condition adopted above.

The situation changes drastically when the string network is intrinsically mirror-noninvariant. The Chern-Simons number,

$$
n_{\mathrm{CS}}=\frac{g^{2}}{8 \pi^{2}} \int d^{3} x \varepsilon_{i j k} A_{i} F_{j k},
$$

is proportional to the helicity of the gauge field. In the case of the string network it was evaluated to be $n_{\mathrm{CS}}=2 \sum_{a<b} L[a, b]+\sum_{a} W[a]$ [9,16, 17], where the first term comes from the linking of any pair of the strings $a, b$ characterized by the linking number $L[a, b]$ [9] while the second one does from the writhing of each individual string $a$ [16, 17] characterized by the writhing number $W[a]$ [20]. The correction to the total action is as in Eq. (1), with $n_{\mathrm{CS}}$ from Eq. (12). To obtain the equation of motion by varying the action over $\mathbf{X}$, one should include the variation of the Chern-Simons number $n_{\mathrm{CS}}$. To this end, the Fourier representation of this number found earlier [17,18,

$$
n_{\mathrm{CS}}=\sum_{a, b} \int d \sigma_{a} d \sigma_{b} \int d^{3} k \exp \left(-i \mathbf{k} \cdot \mathbf{X}_{a b}\right) \frac{i \mathbf{k} \cdot\left[\mathbf{X}_{a}^{\prime}\left(\sigma_{a}\right) \times \mathbf{X}_{b}^{\prime}\left(\sigma_{b}\right)\right]}{\mathbf{k}^{2}}\left(\frac{m_{V}^{2}}{\mathbf{k}^{2}+m_{V}^{2}}\right)^{2},
$$

$\mathbf{X}_{a b} \equiv \mathbf{X}_{a}\left(\sigma_{a}\right)-\mathbf{X}_{b}\left(\sigma_{b}\right)$, is useful. When varying this expression over the string contour $\mathbf{X}$, one should have in mind that the terms with $a \neq b$ vanish, because they contribute to the linking number of any pair of strings which is the topological invariant. The typical term with $a=b$ being the variation of the writhing number $W$ [18], after the integration by parts ?), and dropping the string label $a$, becomes

$$
\begin{aligned}
\delta W= & \int d^{3} k\left(\frac{m_{V}^{2}}{\mathbf{k}^{2}+m_{V}^{2}}\right)^{2} \int d \sigma_{1} d \sigma_{2} \exp \left(-i \mathbf{k} \cdot \mathbf{X}_{12}\right)\left[\delta \mathbf{X}\left(\sigma_{1}\right)-\delta \mathbf{X}\left(\sigma_{2}\right)\right] \\
& \cdot\left[\mathbf{X}^{\prime}\left(\sigma_{1}\right) \times \mathbf{X}^{\prime}\left(\sigma_{2}\right)\right],
\end{aligned}
$$

where $\mathbf{X}_{12} \equiv \mathbf{X}\left(\sigma_{1}\right)-\mathbf{X}\left(\sigma_{2}\right), \sigma_{1,2}$ refers now to the same contour. Since

$$
\int \frac{d^{3} k}{(2 \pi)^{3}}\left(\frac{m_{V}^{2}}{\mathbf{k}^{2}+m_{V}^{2}}\right)^{2} \exp (-i \mathbf{k} \cdot \mathbf{X})=\frac{m_{V}^{3}}{8 \pi} \exp \left(-m_{V}|\mathbf{X}|\right)
$$

\footnotetext{
${ }^{2}$ The surface terms vanish identically for the closed string, and can be made vanishing by imposing the condition of periodicity in the case of the open string.
} 
the contribution of the remote arclength segments is suppressed exponentially with the distance between them, and one may use the expansion $\mathbf{X}\left(\sigma_{2}\right)=\mathbf{X}\left(\sigma_{1}\right)+\mathbf{X}^{\prime}\left(\sigma_{1}\right)\left(\sigma_{2}-\sigma_{1}\right)+\cdots$ up to the third order, to integrate over $z=\sigma_{2}-\sigma_{1}$. One obtains

$$
\delta W=\frac{m_{V}^{3}}{8 \pi} \int d \sigma \delta \mathbf{X} \cdot\left[\mathbf{X}^{\prime} \times \mathbf{X}^{\prime \prime \prime}\right] \int_{-\infty}^{\infty} d z\left(-z^{2}\right) \exp \left(-m_{V}|z|\right)=\frac{1}{2 \pi} \int d \sigma \delta \mathbf{X} \cdot\left[\mathbf{X}^{\prime} \times \mathbf{X}^{\prime \prime \prime}\right]
$$

The variation of the Chern-Simons number of a single string reduces to the variation of the writhing number (15). Then the equation of motion obtained upon the variation of the total action, becomes

$$
\ddot{\mathbf{X}}-\mathbf{X}^{\prime \prime}-\frac{\mu}{4 \pi \varepsilon_{\mathrm{v}}}\left[\mathbf{X}^{\prime} \times \mathbf{X}^{\prime \prime \prime}\right]=0 .
$$

The effect of a nonzero fermionic number characterized by nonzero $\mu$, on the string motion is essentially three- dimensional and is possible only for parity-non-invariant contours. It manifests as an additional force perpendicular to both the tangent, $\mathbf{X}^{\prime}$, and normal, $\mathbf{n}$, vectors of the string. Indeed, using the Frenet equations,

$$
\mathbf{X}^{\prime \prime}=\kappa \mathbf{n}, \mathbf{n}^{\prime}=-\kappa \mathbf{X}^{\prime}+\tau \mathbf{b}, \mathbf{b}^{\prime}=-\tau \mathbf{n},
$$

where $\kappa, \tau$, and $\mathbf{b}$ are, respectively, the curvature, the torsion, and the binormal vector of a curve, one can represent Eq. (16) in the form

$$
\ddot{\mathbf{X}}=\kappa\left(1-\frac{\tau \mu}{4 \pi \varepsilon_{\mathrm{v}}}\right) \mathbf{n}+\frac{\kappa^{\prime} \mu}{4 \pi \varepsilon_{\mathrm{v}}} \mathbf{b} .
$$

The first term on the right hand side is the usual one originating from the tension force but corrected for the interaction with the fermionic matter. The second term is completely new. This term, together with the above correction to the tension, are the classical manifestations of the purely quantum phenomenon of an anomaly. As is argued in Ref. [15], this is due to the appearance of new fermionic levels from the Dirac sea upon applying an external gauge field, in the present case, the variable gauge field of the moving string segment. Note that the time derivative of the writhing number, $\dot{W}[a]=\int d \sigma \dot{\mathbf{X}} \cdot\left[\mathbf{X}^{\prime} \times \mathbf{X}^{\prime \prime \prime}\right] / 2 \pi$, vanishes for the translational motion of the string [18]. Hence, to first order in chemical potential, only the dynamical internal motion of the parity-non-invariant gauge string is sensitive to the external uniform background density of fermions. Note the difference of an additional force in the equation of motion (16) with another one obtained in Ref. [21]. An additional force besides the tension found there depends on the string local velocity and in this sense is anologous to the known Magnus force acting even on the straight string. In our case, the force is of purely chiral origin. It is independent of the local velocity, but acts only on the strings with the mirror-non-invariant contour.

Leaving the time-dependent configurations for a future work, let us find the static solutions to Eq. (16) and perform their stability analysis. As is evident from Eq. (18), there are two static solutions. The first solution is $\kappa=0, \kappa^{\prime}=0$ which means the straight line, say, $\mathbf{X}(\sigma)=\sigma \mathbf{e}_{z}$. The second one is $\kappa^{\prime}=0, \tau=4 \pi \varepsilon_{\mathrm{v}} / \mu$, which means a curve with the constant curvature $\kappa$ and torsion $\tau$. An explicit form of the contour in this case that can be found from solving the Frenet equations (17), is a helix, 


$$
\mathbf{X}(\sigma)=R\left[\mathbf{e}_{x} \cos \left(\frac{\sigma}{a}\right)+\mathbf{e}_{y} \sin \left(\frac{\sigma}{a}\right)\right]+\mathbf{e}_{z} \frac{l \sigma}{2 \pi a}
$$

whose radius $R$ and step $l$ are not arbitrary but subjected to the condition

$$
l / 2 \pi a^{2}=4 \pi \varepsilon_{\mathrm{v}} / \mu .
$$

Hereafter we denote $a=\sqrt{R^{2}+(l / 2 \pi)^{2}}$. As usual, the stability analysis demands the study of the negative modes of the second variational derivative of the energy functional. Using the expression for $\delta W$ (14) to obtain the second variational derivative of this functional in the form

$$
\frac{\delta^{2} E}{\delta X_{i}(\sigma) \delta X_{j}\left(\sigma^{\prime}\right)}=-\left[2 \varepsilon_{\mathrm{v}} \delta_{i j} \partial_{\sigma}^{2}+\mu \varepsilon_{i j k}\left(2 X_{k}^{\prime \prime \prime} \partial_{\sigma}+3 X_{k}^{\prime \prime} \partial_{\sigma}^{2}+X_{k}^{\prime} \partial_{\sigma}^{3}\right) / 2 \pi\right] \delta\left(\sigma-\sigma^{\prime}\right),
$$

one gets, after the integration by parts (see footnote 2),

$$
\delta^{2} E=\varepsilon_{\mathrm{v}} \int d \sigma\left(\boldsymbol{\xi}^{\prime 2}+\mu \mathbf{X}^{\prime} \cdot\left[\boldsymbol{\xi}^{\prime} \times \boldsymbol{\xi}^{\prime \prime}\right] / 4 \pi\right)
$$

where $\mathbf{X}, \boldsymbol{\xi}$ are, respectively, the unperturbed contour and its variation. The latter should obey the condition

$$
\xi^{\prime} \cdot \mathbf{X}^{\prime}=0
$$

in order to preserve the gauge choice $\mathbf{X}^{\prime 2}=1$ of the static problem. First, consider the straight line solution. Then Eq. (23) reduces to $\xi_{3}^{\prime}=0$, and the second variation of the energy functional becomes

$$
\begin{aligned}
\delta^{2} E= & \varepsilon_{\mathrm{v}} \int d \sigma\left[\xi_{1}^{\prime 2}+\xi_{2}^{\prime 2}+\frac{\mu}{4 \pi \varepsilon_{\mathrm{v}}}\left(\xi_{1}^{\prime} \xi_{2}^{\prime \prime}-\xi_{2}^{\prime} \xi_{1}^{\prime \prime}\right)\right] \\
& =\varepsilon_{\mathrm{v}} \sum_{n=1}^{\infty}\left(\xi_{1 n}^{2}+\xi_{2 n}^{2}+\frac{\mu n}{\varepsilon_{\mathrm{v}} L} \xi_{1 n} \xi_{2 n}\right)\left(\frac{2 \pi n}{L}\right)^{2},
\end{aligned}
$$

where the use is made of an expansion of $\boldsymbol{\xi}$ into the normal modes

$$
\boldsymbol{\xi}(\sigma)=\sqrt{2 / L} \sum_{n=1}^{\infty} \boldsymbol{\xi}_{n} \cos (2 \pi n \sigma / L)
$$

found from the demand of the periodicity in $\sigma$ with the period $L$. Diagonalisation of the expression in the second line in Eq. (24) shows that the modes with $n<2 \varepsilon_{\mathrm{v}} L / \mu$ are stable while higher ones are unstable. One should set $L \rightarrow \infty$ for the infinitely straight string, which means that such a string in the fermionic matter is always stable. However, it is known [8] that Z-strings terminating on monopoles may have the finite length. The latter can be chosen such that one may neglect the mass of the monopoles as compared to the total energy of Z-string, in order not to take into account the monopole contribution to the action. Then one comes to the conclusion that sufficiently high modes on the straight Z-string of finite length destabilize it in the presence of the fermionic matter with the nonzero chemical potential. 
The stability analysis of the helical solution (19) can be reduced to the case of the straight string by means of the change of variables. Indeed, $\xi_{3}^{\prime}$ found from Eq. (23) is

$$
\xi_{3}^{\prime}=\frac{2 \pi R}{l}\left(\xi_{1}^{\prime} \sin \frac{\sigma}{a}-\xi_{2}^{\prime} \cos \frac{\sigma}{a}\right) .
$$

Substituting this expression into Eq. (22) and using Eq. (20), one obtains

$$
\begin{aligned}
\delta^{2} E= & \varepsilon_{\mathrm{v}} \int d \sigma\left[\xi_{1}^{\prime 2}+\xi_{2}^{\prime 2}+\left(\frac{2 \pi R}{l}\right)^{2}\left(\xi_{1}^{\prime} \sin \frac{\sigma}{a}-\xi_{2}^{\prime} \cos \frac{\sigma}{a}\right)^{2}+\left(\frac{2 \pi a}{l}\right)^{2} a\left(\xi_{1}^{\prime} \xi_{2}^{\prime \prime}-\xi_{2}^{\prime} \xi_{1}^{\prime \prime}\right)\right. \\
& \left.-\left(\frac{2 \pi R}{l}\right)^{2}\left(\xi_{1}^{\prime} \cos \frac{\sigma}{a}+\xi_{2}^{\prime} \sin \frac{\sigma}{a}\right)^{2}\right]
\end{aligned}
$$

Now, introducing new variables $\Xi_{1,2}$ according to the relation

$$
\left(\begin{array}{l}
\xi_{1}^{\prime} \\
\xi_{2}^{\prime}
\end{array}\right)=\frac{1}{\sqrt{2}}\left(\begin{array}{cc}
\cos \frac{\sigma}{a} & -\sin \frac{\sigma}{a} \\
\sin \frac{\sigma}{a} & \cos \frac{\sigma}{a}
\end{array}\right)\left(\begin{array}{c}
\Xi_{1}^{\prime} \\
\frac{l}{2 \pi a} \Xi_{2}^{\prime}
\end{array}\right),
$$

one finds from Eq. (26) that

$$
\begin{aligned}
\delta^{2} E= & \varepsilon_{\mathrm{v}} \int d \sigma\left[\Xi_{1}^{\prime 2}+\Xi_{2}^{\prime 2}+\frac{\pi a^{2}}{l}\left(\Xi_{1}^{\prime} \Xi_{2}^{\prime \prime}-\Xi_{2}^{\prime} \Xi_{1}^{\prime \prime}\right)\right] \\
& =\varepsilon_{\mathrm{v}} \sum_{n=1}^{\infty}\left(\Xi_{1 n}^{2}+\Xi_{2 n}^{2}+\frac{(2 \pi a)^{2} n}{l L} \Xi_{1 n} \Xi_{2 n}\right)\left(\frac{2 \pi n}{L}\right)^{2}
\end{aligned}
$$

where the expansion into the normal modes analogous to Eq. (25) in the case of the straight string is used. One can see, using Eq. (20), that the stability condition for the helical solution coincides with that in the case of the straight string: the modes with $n<l L / 2 \pi^{2} a^{2}=2 \varepsilon_{\mathrm{v}} L / \mu$ are stable. Again, an infinitely long helix is stable, while the higher modes on the helix of finite length become negative in the presence of the fermionic matter with $\mu \neq 0$.

To conclude, two new effects due to the presence of fermionic matter with finite density important for the gauge string configurations are found. First, a nonzero magnetic-like helicity is induced on such a non-chiral object as the straight string. Second, the fermionic background exerts an influence on the motion of the string with intrinsically parity-noninvariant contours as an additional force besides the tension. The correction to the string equation of motion found here should be compared with the lowest order finite thickness correction, $\sim\left(\kappa / m_{H}\right)^{2}$, with $\kappa$ being the curvature of the contour, calculated in [12, 13. Taking, for instance, the case of the helical contour of the step $l$ one can find the condition of the dominance of the $\mu \neq 0$ correction in the form $\mu l \gg \lambda^{-2} \ln \lambda$, where the self-interaction coupling of the Higgs field is large, $\lambda^{2} \gg 1$, in the London limit adopted here. Static solutions corresponding to the balance of the above forces are found, and their stability is explored. The stability refers to that in the background of fermions. If one has in mind the electroweak Z-string [8] that definitely contributes to the anomaly of the sum of the baryon and lepton currents, it possesses its own instabilities, see recent review [22]. The question of whether these instabilities can be neutralized or not is still an open one [22]. Despite this, the Abelian-like spontaneously broken gauge model of the vector field coupled to the chiral fermions considered in the present work can be viewed as an ingredient of some wider theoretical framework and by this reason might be of interest as an additional demonstration of how purely quantum phenomenon of an anomaly manifests on the macroscopic scale. 


\section{REFERENCES}

[1] A. D. Linde, Phys. Lett. 86B, (1979) 39.

[2] B. Bajc, A. Riotto, G. Senjanović, Phys. Rev. Lett. 81, (1998) 1355; Mod. Phys. Lett. A13, (1998) 2955.

[3] M. Joyce, M. E. Shaposhnikov, Phys. Rev. Lett. 79, (1997) 1193;

M. Giovannini, M. E. Shaposhnikov, Phys. Rev. D57, (1998) 2186.

[4] F. Freese, E. W. Kolb, M. S. Turner, Phys. Rev. D27, (1983) 1689;

H. -S. Kang, G. Steigman, Nucl. Phys. B372, (1992) 494.

[5] A. Vilenkin, E. P. S. Shellard, Cosmic Strings and Other Topological Defects (Cambridge University Press, 1994).

[6] M. B. Hindmarsh, T. W. B. Kibble, Rep. Progr. Phys. 58, (1995) 477.

[7] A. A. Abrikosov, ZhETF 32, (1957) 1441;

H. B. Nielsen, P. Olesen, Nucl. Phys. 61, (1973) 45.

[8] Y. Nambu, Nucl. Phys. B130, (1977) 505;

N. S. Manton, Phys. Rev. D28, (1983) 2019;

T. Vachaspati, Phys. Rev. Lett. 68, (1992) 1977, 69, (1992) 216(E).

[9] T. Vachaspati, G. B. Field, Phys. Rev. Lett. 73, (1994) 373.

[10] D. Förster, Nucl. Phys. B81, (1974) 84.

[11] P. Orland, Nucl. Phys. B428, (1994) 221.

[12] H. Arodź, Nucl. Phys. B450, (1995) 189.

[13] M. Anderson, F. Bonjour, R. Gregory, J. Stewart, Phys. Rev. D56, (1997) 8014.

[14] A. N. Redlich, L. C. R. Wijewardhana, Phys. Rev. Lett. 54, (1985) 970.

[15] V. A. Rubakov, A. N. Tavkhelidze, Theor. Math. Phys. 65, (1985) 250.

[16] M. Sato, S. Yahikozawa, Nucl. Phys. B436, (1995) 100.

[17] A. A. Kozhevnikov, Phys. Rev. D52, (1995) 6053.

[18] A. A. Kozhevnikov, Phys. Rev. D59, (1999) 085003, hep-ph/9812512.

[19] G. Bimonte, G. Lozano, Phys. Lett. B348, (1995) 457.

[20] H. K. Moffatt, Renzo L. Ricca, Proc. R. Soc. London, A439, (1992) 411.

[21] Uri Ben-Ya'cov, Phys. Rev. D51, (1995) 4561.

[22] A. Achúcarro and T. Vachaspati, "Semilocal and Electroweak Strings", hep-ph/9904229. 